\title{
About the translators
}

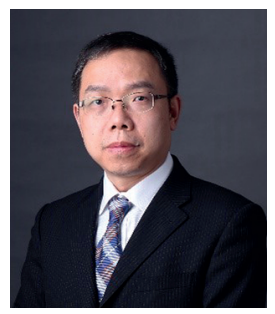

\section{Professor Zhuguo LI}

School of Materials Science and Engineering

Shanghai Jiao Tong University

Professor Zhuguo LI has obtained his bachelor degree in welding and automation in 1994 from Shanghai Jiao Tong University, master's degree in welding engineering in 1997 from the same university, and $\mathrm{PhD}$ in materials processing science from Osaka University in 2004. Since then, he joined Shanghai Jiao Tong University. He became a full-time professor since 2010 and a distinguished professor since 2020. Now he is the vice dean of School of Materials Science and Engineering, Shanghai Jiao Tong University, and the director of Shanghai Key Laboratory of Materials Laser Processing and Modification. He also serves as the deputy secretary general of China Welding Society. His research interests include laser welding, laser cladding, and laser additive manufacturing, and he is the coauthor of more than $150 \mathrm{SCI}$ papers published on peer-review journals. He won the first prize of Science and Technology Award from CMES in 2016, and the first prize of Science and Technology Progress Award from Shanghai Municipality in 2015 and 2019.

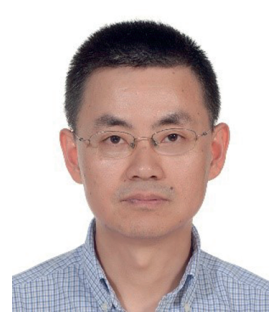

Professor Da Shu is currently the deputy director of Shanghai Key Laboratory of Advanced High-Temperature Materials and Precision Forming, School of Materials Science and Engineering, Shanghai Jiao Tong University (SJTU). He received his BSc (1994) at Nanjing University of Aeronautics and Astronautics and his MSc (1997) and PhD (2001) at SJTU. His subsequent career has been a postdoc (2001), a lecturer (2003), an associate professor (2007), and a professor (2013) at SJTU. He was an academic visitor at the Department of Materials, Oxford University, from 2009 to 2010 . His research focuses on processing of advanced metallic materials and control of solidified structure. He has published more than 100 peer-reviewed articles and patented 48 inventions (including one US patent). He has also won numerous awards, including (1) the second prize of National Technology Invention Awards in 2018 and 2006, (2) Rising Star of Shanghai Municipal Science and Technology Commission in 2007, (3) the second prize of Science and Technology Awards of China Non-Ferrous Metals Industry Association in 2008, and (4) the New-Century Talents of Ministry of Education in 2013.

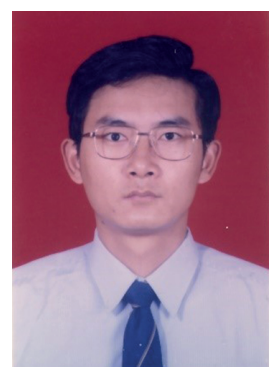

Zhenghong Guo is an associate professor at the School of Materials Science and Engineering, Shanghai Jiao Tong University, where he has been a faculty member since 2001 . From 2002, he has been taking charge of several courses for students, mainly national excellent course "The Fundamentals of Materials Science" for undergraduate students and school-level honor course "Principle of Phase Transformation" for graduate students.

Guo earned his doctoral degree in materials science at Shanghai Jiao Tong University. His research interests lie in the area of microstructural control by solid-state transformation, covering theoretical exploration to novel steel development. 


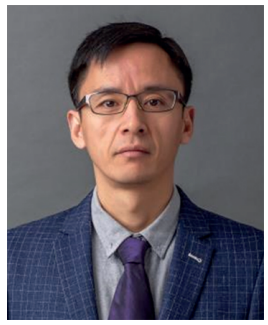

Xiaodong Wang is an associate professor at the School of Materials Science and Engineering, Shanghai Jiao Tong University. For a long time, he taught the National Excellent Course "Fundamentals of Materials Science" for undergraduates and served as the responsible professor of the core course "Microscopy and Spectroscopic Characterization" for postgraduates. He studied modestly and was good at thinking. He actively explored the curriculum teaching reform and put it into practice, and finally formed his own unique teaching concept and teaching method, which is popular and well received by students. He won the second prize of individual award of "Teaching and Education Award," the fifth "Excellent Teaching Award" of Shanghai Jiao Tong University, and "the most popular teacher" award of School of Materials Science and Engineering. He cares for students and encourages them to be honest and innovative. He earnestly fulfills the primary duty of teaching and educating.

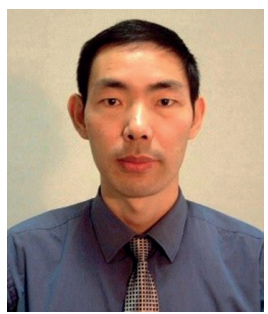

Guo He is currently a professor of materials science and engineering at Shanghai Jiao Tong University (Shanghai, China). He received his PhD in materials science from Northwestern Polytechnical University (Xian, China) in 1994, MS in materials science from Xi'an Jiao Tong University (Xian, China) in 1991, and BS in materials science and engineering from Hefei University of Technology (Hefei, China) in 1983. From 1994, he served as a lecturer and an associate professor successively at the University of Science and Technology Beijing (Beijing, China). In 2000, he moved to City University of Hong Kong (Hong Kong, China) as a visiting scientist at the Department of Physics and Materials Science. Later, he worked at Leibniz Institute for Solid State and Materials Research Dresden (Dresden, Germany) as an Alexander-von-Humboldt research fellow from 2001 to 2003, then, he moved to National Institute for Materials Science (Tsukuba, Japan) as a visiting scientist. In 2005, he joined the Faculty of Shanghai Jiao Tong University as a professor. His research interests focus on porous titanium, nanostructured titanium, and bioactive coating or surface modification of titanium for orthopedic applications. He has published hundreds of peer-reviewed journal papers and more than a dozen patents. His papers have been cited thousands of times. In recent years, he has devoted himself to the development of bioceramic materials and the application of the porous titanium technology that is being developed in his lab. 